Оригинальная статья/Original article

УДК 330

DOI: http://doi.org/10.20914/2310-1202-2017-2-290-295

Математическое моделирование показателей развития региона в
концептуальной модели оценки влияния внешней среды на
региональную социально-экономическую систему

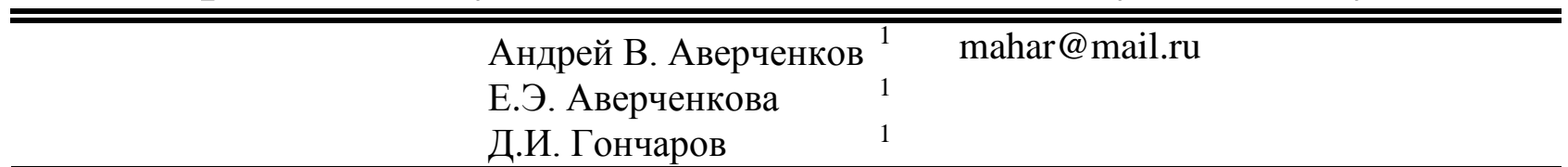

$\mathbf{1}$ Брянский государственный технический университет, бул. 50-лет Октября, 7, г. Брянск, 241035, Россия

Реферат. В статье рассмотрены направления расширения комплексного подхода к оценке влияния внешней среды на региональную социально-экономическую систему. Авторами предлагается подход, основанный на математическом моделировании основных показателей развития региона. Эмпирическими данными для разработки моделей стала ведомственная структура расходов Брянской области департамента экономического развития и показатели, представляемые для разработки прогноза социально-экономического развития Российской Федерации на период до 2019 года. При формировании модели показателей социально-экономического развития региона были получены аппроксимирующие функции, ограниченные диапазоном заданных значений ведомственных расходов бюджета Брянской области за 2012-2019 гг. и выступающих в качестве интерполирующей зависимости. Для оптимального подбора параметров уравнений был использован метод наименьших квадратов. Практическая реализация концептуальной модели оценки влияния внешней среды на региональную социально-экономическую систему определяет необходимость ее автоматизации. Разрабатываемая на основе представленной модели информационная советующая система позволит автоматизировать процесс поддержки принятия управленческого решения. Полученная модель оценки влияния внешней среды на региональную социально-экономическую систему представляет собой информационно-аналитическую систему отслеживания региональной ситуации как основы для последующего принятия управленческих решений.

Ключевые слова: региональная социально-экономическая система, прогноз показателей развития, математическое моделирование

\title{
Mathematical modeling of regional development indicators in a conceptual model for assessing the impact of the external environment on the regional socio-economic system
}

\begin{tabular}{lll}
\hline \hline Andrew V. Averchenkov & 1 & mahar@mail.ru \\
E.Je. Averchenkova & 1 & \\
D.I. Goncharov & 1 & \\
\hline
\end{tabular}

1 Bryansk State Technical University, Bulvar 50-letiya Oktyabrya, 7, Bryansk, 241035, Russia
Summary. In the article the directions of expansion of the complex approach to the assessment of the influence of the external
environment on the regional socio-economic system are considered. The authors propose an approach based on mathematical
modeling of the main indicators of the development of the region. Empirical data for the development of models has become the
departmental expenditure structure of the Bryansk region of the Department of Economic Development and the indicators presented
for the development of the forecast of the socio-economic development of the Russian Federation for the period until 2019. When
forming a model of indicators of socio-economic development of the region, approximating functions were obtained, limited by
the range of specified values of departmental expenditures of the budget of the Bryansk region for 2012-2019. And acting as an
interpolating relationship. For the optimal selection of the parameters of the equations, the method of least squares was used.
Practical implementation of the conceptual model for assessing the impact of the external environment on the regional socio-
economic system determines the need for its automation. The information advisory system developed on the basis of the presented
model will allow to automate the process of supporting the adoption of the management decision. The obtained model for assessing
the influence of the external environment on the regional socio-economic system is an information and analytical system for
monitoring the regional situation as the basis for subsequent management decisions. Keywords: regional socio-economic system, forecast of development indicators, mathematical modeling

Keywords: regional socio-economic system, forecast of development indicators, mathematical modeling

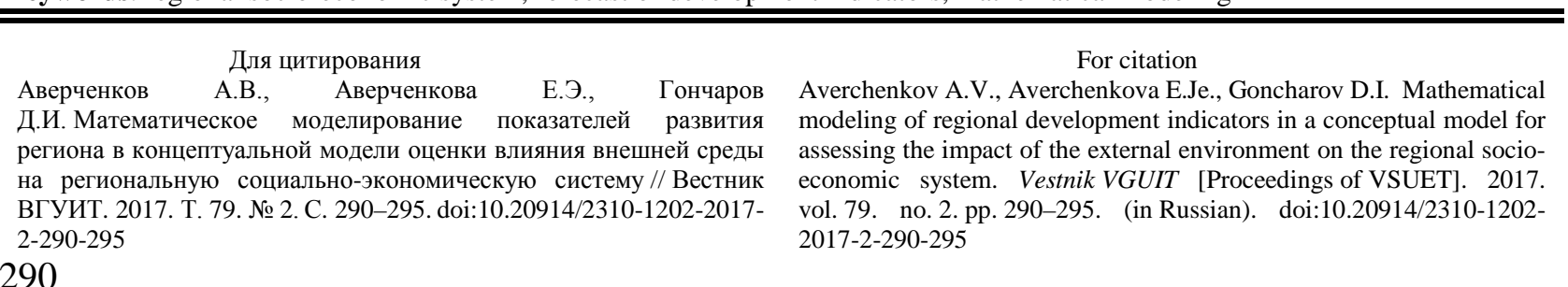




\section{Введение}

Ранее в работах авторов $[4,5,7]$ было показано, что определить влияние внешней среды на текущее состояние региональной социально-экономической системы можно с помощью комплексного подхода. Так, были описаны возможности экспертного оценивания изучаемого влияния и мониторинговые оценки с использованием внешних статистических данных. Однако представляется важным, что для повышения достоверности комплексного подхода к изучению влияния внешней среды на регион, использовать методы математического моделирования основных показателей его социально-экономического развития.

Вклад в осознание особенностей процессов прогнозирования социально-экономических показателей развития региона внесли отечественные ученые-практики, в том числе Е.В. Бережная, В.Н. Бурков, С.Ю. Глазьев, Т.А. Дуброва, В.В. Егоров, А.Ю. Заложнев, С.В. Леонтьев, Д.А. Новиков, А.Д. Настенко, А.В. Цветков, Т.Е. Шохина. В их работах показано, что анализ и прогнозирование социально-экономического развития субъектов РФ представляется отправной точкой работы при решении задач по управлению устойчивым развитием регионов.

Цель работы - расширение концептуальной модели оценки влияния внешней среды на регион посредством разработки конкретных математических моделей, определяющих социально-экономическое развитие Брянской области.

\section{Эмпирическая база исследования}

Для формирования перечня показателей социально-экономического развития Брянского региона был использован список закрепленных показателей прогноза социально-экономического развития Брянской области за исполнительными органами государственной власти Брянской области и структурными подразделениями администрации Брянской области [3]. В качестве базового структурного подразделения в данной работе был использован департамент экономического развития Брянской области и показатели, закрепленные за ним, по следующим направлениям: население, производство товаров и услуг, торговля и услуги населению, внешнеэкономическая деятельность региона, малое и среднее предпринимательство, инвестиции региона, труд и занятость, развитие социальной сферы, окружающая среда, туризм.
Такой выбор объясняется тем, что департамент экономического развития Брянской области реализует задачи по социально-экономическому развитию региона, осуществляет стратегическое планирование, способствует развитию инновационной активности в регионе, осуществляет контроль выполнения государственных и региональных программ на территории Брянской области и т. д. [8]. Отсюда, показатели, определяемые этим департаментом, имеют ключевое место в социально-экономическом развитии региона.

В работе предлагается сформировать прогноз развития региона по данным направлениям, общее количество конкретных показателей к расчету составляет 44. При формировании математических функций были использованы статистические данные за 2011-2016 гг. и прогнозируемые данные Брянского региона до 2019 г., взятые из официальной документации Правительства Брянской области для разработки прогноза социально-экономического развития РФ на период до 2019 года (для субъектов РФ) [2].

Отметим, что данные показатели являются основными макроэкономическими показателями социально-экономического развития Брянской области за предыдущие годы, а также учитывают сценарные условия развития экономики Российской Федерации на 2017-2019 годы. Кроме того, эти показатели учитывают приоритеты и целевые индикаторы, определенные в Стратегии социально-экономического развития Брянской области на период до 2025 года, указах Президента РФ от 7 мая 2012 года № 596-606, государственных программах Брянской области, а также ход реализации мероприятий по обеспечению устойчивого развития экономики и социальной стабильности Российской Федерации и Брянской области.

При формировании предлагаемых моделей показателей развития Брянского региона был использован такой влияющий фактор, как финансирование соответствующих направлений социально-экономического развития региона. Ранее в статьях авторов были рассмотрены особенности формирования и расходования бюджета Брянской области на 2016-2019 и 2013-2015 гг. [6]. Так, было показано, что ведомственная структура расходов бюджета как распределение бюджетных ассигнований, предусмотренных законом Брянской области о бюджете [1], определяет объем и направление 
финансирования приоритетных для региона направлений его социально-экономического развития. Как отмечалось выше, в качестве базового структурного подразделения в данной работе был использован департамент экономического развития Брянской области, поэтому при формировании моделей была использована ведомственная структура расходов Брянской области за 2011-2019 гг. на его примере.

\section{Моделирование показателей прогноза социально-экономического развития Брянской области}

При формировании модели показателей социально-экономического развития региона были получены аппроксимирующие функции, ограниченные диапазоном заданных значений ведомственных расходов бюджета Брянской области за 2012-2019 гг. и выступающих в качестве интерполирующей зависимости. Для оптимального подбора параметров уравнений был использован метод наименьших квадратов.

Сложность, взаимное влияние процессов, протекающих в социально-экономической с истеме региона, определило выбор типа аппроксимации с помощью полинома 4-й или 5-й степени. Формирование функций производилось с помощью ресурсов MS Excel. Результатом модели является значение показателей развития социально-экономичского региона в виде функции от объема ведомственных расходов области. Каждая функция будет характеризоваться величиной достоверности аппроксимации $R_{2}$, определяющей степень соответствия трендовой модели исходным данным. Использование критерия Фишера для проверки значимости построенной регрессионной модели оценивалось с надежностью 95\%. Критическое (табличное) значение критерия Фишера составило $F_{\text {кр }}=5,99$, с ним сравнивались расчетные значения $F_{\text {расч}}$, которые для всех функций были одинаковым, так как количество наблюдений $(n=8$ «лет») и число объясняющих переменных $(k=1)$ во всех случаях было одинаковым. Аппроксимирующие функции по основным показателям развития региональной социальноэкономической системы и их расчетные значения $F$-критерия представлены в таблице 1.

Очевидно, что значения достоверности аппроксимации $R_{2}$ для всех рассчитанных функций имеют величину, превосходящую критическое значение, равное 0,5. Это позволяет сделать вывод о том, что построенные модели прогнозирования основных показателей развития региональной социально-экономической системы пригодны для дальнейшего анализа.
Все расчетные значения $F$-критерия больше табличного критического значения. Значит, с вероятностью 95\% полученные модели являются адекватными.

\section{Формирование комплексного оценки влияния внешней среды на региональную социально-экономическую систему}

Актуальность формирования методологии комплексного подхода к оценке влияния внешней среды на региональную социально-экономическую систему обосновывается необходимостью обеспечения динамичности и объективности оценки происходящих в ней изменений. Это обеспечивается учетом следующих четырех позиций:

1. Анализируются внешние источники информации о состоянии региона.

2. Производится градация текущего состояния регионального промышленного комплекса.

3. Проводится экспертная оценка влияния внешней среды на регион.

4. Формируются прогнозные значения основных показателей развития региона, отслеживается их тренд.

Обобщение результатов исследований, представленных в работах [4-6] позволило сформировать следующую концептуальную модель оценки влияния внешней среды на региональную социально-экономическую систему (рисунок 1).

В модели представлены четыре основных блока:

- информационная система мониторинга внешней среды региональной социальноэкономической системы;

- мониторинг состояния регионального промышленного комплекса;

- экспертная оценка влияния внешней среды на региональную социально-экономическую систему;

- прогноз изменения основных показателей развития региона на основе применения методов математического моделирования.

Bce четыре блока независимы друг от друга и являются основанием для принятия управленческих решений, обеспечивающих нивелирование или усиление влияния внешней среды на региональную социально-экономическую систему.

Практическая реализация концептуальной модели оценки влияния внешней среды на региональную социально-экономическую систему определяет необходимость ее автоматизации. Разрабатываемая на основе представленной модели информационная советующая система позволит автоматизировать процесс поддержки принятия управленческого решения. 
Таблица 1.

Некоторые функций аппроксимаций основных показателей развития региональной социально-экономической системы (на примере Брянской области)

Some functions of approximations of the main indicators of the development of the regional socio-economic system (on the example of the Bryansk region)

\begin{tabular}{|c|c|c|c|c|}
\hline $\begin{array}{l}\text { Частные показатели прогноза социально-экономического развития региона } \\
\text { Particular indicators of socio-economic development forecast for the region }\end{array}$ & $\begin{array}{l}\text { Размерность показателя } \\
\text { Dimension of the indicator }\end{array}$ & $\begin{array}{c}\text { Аппроксимирующая функция } \\
\text { Approximating function }\end{array}$ & $\begin{array}{c}\text { Показатель достовер- } \\
\text { ности аппроксимации } \\
\text { Indicator of reliability } \\
\text { of approximation } \mathrm{R}^{2} \\
\end{array}$ & $\begin{array}{c}\text { Критерий } \\
\text { Фишера } \\
\text { F-criteria }\end{array}$ \\
\hline \multicolumn{5}{|c|}{\begin{tabular}{|l|l|} 
Производство товаров и услуг & Production of goods and services \\
\end{tabular}} \\
\hline $\begin{array}{l}\text { Выпуск товаров и услуг } \\
\text { Release of goods and services }\end{array}$ & $\begin{array}{l}\text { MлH pyб. } \\
\text { mln RUB }\end{array}$ & $\begin{array}{c}\mathrm{y}=-262 \mathrm{x}^{4}+4653,6 \mathrm{x}^{3}- \\
23554 \mathrm{x}^{2}+63994 \mathrm{x}+368926\end{array}$ & 0,9935 & 917,08 \\
\hline $\begin{array}{l}\text { Индекс промышленного производства } \\
\text { Index of industrial production }\end{array}$ & \begin{tabular}{c|} 
\% к предыдущему году \\
в сопоставимых ценах \\
\% To the previous year in \\
comparable prices
\end{tabular} & $\begin{array}{c}y=0,2975 x^{4}-5,686 x^{3}+ \\
36,884 x^{2}-91,942 x+175,52\end{array}$ & 0,8304 & 29,38 \\
\hline \multicolumn{5}{|c|}{ Торговля и услуги населению| Trade and services to the population } \\
\hline $\begin{array}{l}\text { Индекс потребительских цен за период с начала года } \\
\text { Consumer price index for the period from the beginning of the year }\end{array}$ & $\begin{array}{l}\text { к соответствующему пери- } \\
\text { оду предыдущего года, \% } \\
\text { To the corresponding period } \\
\text { of the previous year, \% }\end{array}$ & $\begin{array}{c}\mathrm{y}=0,0811 \times 4-1,2929 \times 3+ \\
6,0538 \times 2-7,5484 \times+107,64\end{array}$ & 0,7279 & 16,05 \\
\hline $\begin{array}{l}\text { Oборот розничной торговли } \\
\text { Retail trade turnover }\end{array}$ & $\begin{array}{l}\text { Mлн pyб. } \\
\text { mln RUB }\end{array}$ & $\begin{array}{c}\mathrm{y}=81,634 \times 4-1074,6 \times 3+ \\
2740,1 \times 2+22039 \mathrm{x}+127823\end{array}$ & 0,9917 & 716,89 \\
\hline \multicolumn{5}{|c|}{ Внешнеэкономическая деятельность | Foreign economic activity } \\
\hline $\begin{array}{l}\text { Экспорт товаров } \\
\text { Export }\end{array}$ & \begin{tabular}{c|} 
млн долл. США \\
mln USD
\end{tabular} & $\begin{array}{c}\mathrm{y}=0,113 \mathrm{x}^{4}-3,3934 \mathrm{x}^{3}+ \\
35,592 \mathrm{x}^{2}-149,51 \mathrm{x}+533,43\end{array}$ & 0,9216 & 70,53 \\
\hline $\begin{array}{l}\text { Импорт товаров } \\
\text { Import }\end{array}$ & $\begin{array}{l}\text { млн долл. США } \\
\text { mln USD }\end{array}$ & $\begin{array}{c}\mathrm{y}=-3,8672 \mathrm{x}^{4}+72,412 \mathrm{x}^{3}- \\
434,44 \mathrm{x}^{2}+822,12 \mathrm{x}+789,48\end{array}$ & 0,9791 & 281,08 \\
\hline \multicolumn{5}{|c|}{$\begin{array}{l}\text { Малое и среднее предпринимательство, включая микропредприятия } \\
\text { Small and medium enterprises, including microenterprises }\end{array}$} \\
\hline $\begin{array}{l}\text { Количество предприятий } \\
\text { Count }\end{array}$ & $\begin{array}{c}\text { единиц } \\
\text { units }\end{array}$ & $\begin{array}{c}y=-0,589 x^{4}+13,739 x^{3}- \\
136,41 x^{2}+769,38 x+10859\end{array}$ & 0,9799 & 292,51 \\
\hline \multicolumn{5}{|c|}{\begin{tabular}{l|l|l} 
Инвестиции & Investments \\
\end{tabular}} \\
\hline $\begin{array}{l}\text { Инвестиции в основной капитал } \\
\text { Investments in fixed assets }\end{array}$ & $\begin{array}{l}\text { млн pyб. } \\
\text { mln RUB }\end{array}$ & $\begin{array}{l}y=-170,57 x^{4}+3407,3 x^{3}- \\
23172 x^{2}+64250 x+1868,7\end{array}$ & 0,9883 & 506,82 \\
\hline \multicolumn{5}{|c|}{\begin{tabular}{ll|l} 
& Труд и занятость | Work and employment \\
\end{tabular}} \\
\hline $\begin{array}{l}\text { Среднемесячная номинальная начисленная з/п в целом по региону } \\
\text { Average monthly nominal accrued wages in the region as a whole }\end{array}$ & $\begin{array}{l}\text { тыс. pyб. } \\
\text { thousand RUB }\end{array}$ & $\begin{array}{c}\mathrm{y}=-0,0061 \mathrm{x}^{4}+0,1503 \mathrm{x}^{3}- \\
1,2889 \mathrm{x}^{2}+5,5514 \mathrm{x}+12,082\end{array}$ & 0,9988 & 4994,00 \\
\hline \multicolumn{5}{|c|}{ Развитие социальной сферы | Development of the social sphere } \\
\hline $\begin{array}{l}\text { Численность обучающихся общеобразовательных учреждениях (без вечерних } \\
\text { (сменных) общеобразовательных учреждениях (на начало учебного года) } \\
\text { The number of students of general education institutions (without evening (shift) } \\
\text { general education institutions (at the beginning of the academic year) }\end{array}$ & $\begin{array}{l}\text { тыс. чел. } \\
\text { thousand people }\end{array}$ & $\begin{array}{c}y=0,0319 x^{4}-0,6447 x^{3}+ \\
4,2501 x^{2}-8,8019 x+117,88\end{array}$ & 0,9985 & 3994,00 \\
\hline \multicolumn{5}{|c|}{\begin{tabular}{ll|l} 
& Окружающая среда & Environment
\end{tabular}} \\
\hline $\begin{array}{l}\text { Текущие затраты на охрану окружающей среды } \\
\text { Current costs of protecting the environment }\end{array}$ & $\begin{array}{l}\text { в ценах соответствующих } \\
\text { лет; млн руб. } \\
\text { In the prices of the correspond- } \\
\text { ing years; Million RUB. }\end{array}$ & $\begin{array}{l}y=1,1495 x^{4}-19,906 x^{3}+ \\
101,62 x^{2}-86,73 x+479,17\end{array}$ & 0,9782 & 269,23 \\
\hline \multicolumn{5}{|c|}{\begin{tabular}{|l|l|} 
Tуризм & Tourism \\
\end{tabular}} \\
\hline $\begin{array}{l}\text { Численность российских граждан, выехавших за границу } \\
\text { Number of Russian citizens traveling abroad }\end{array}$ & $\begin{array}{c}\text { тыс. чел. } \\
\text { thousand people }\end{array}$ & $\begin{array}{l}y=-0,1417 x^{4}+2,5657 x^{3}- \\
14,695 x^{2}+24,86 x+33,571\end{array}$ & 0,9413 & 96,21 \\
\hline
\end{tabular}




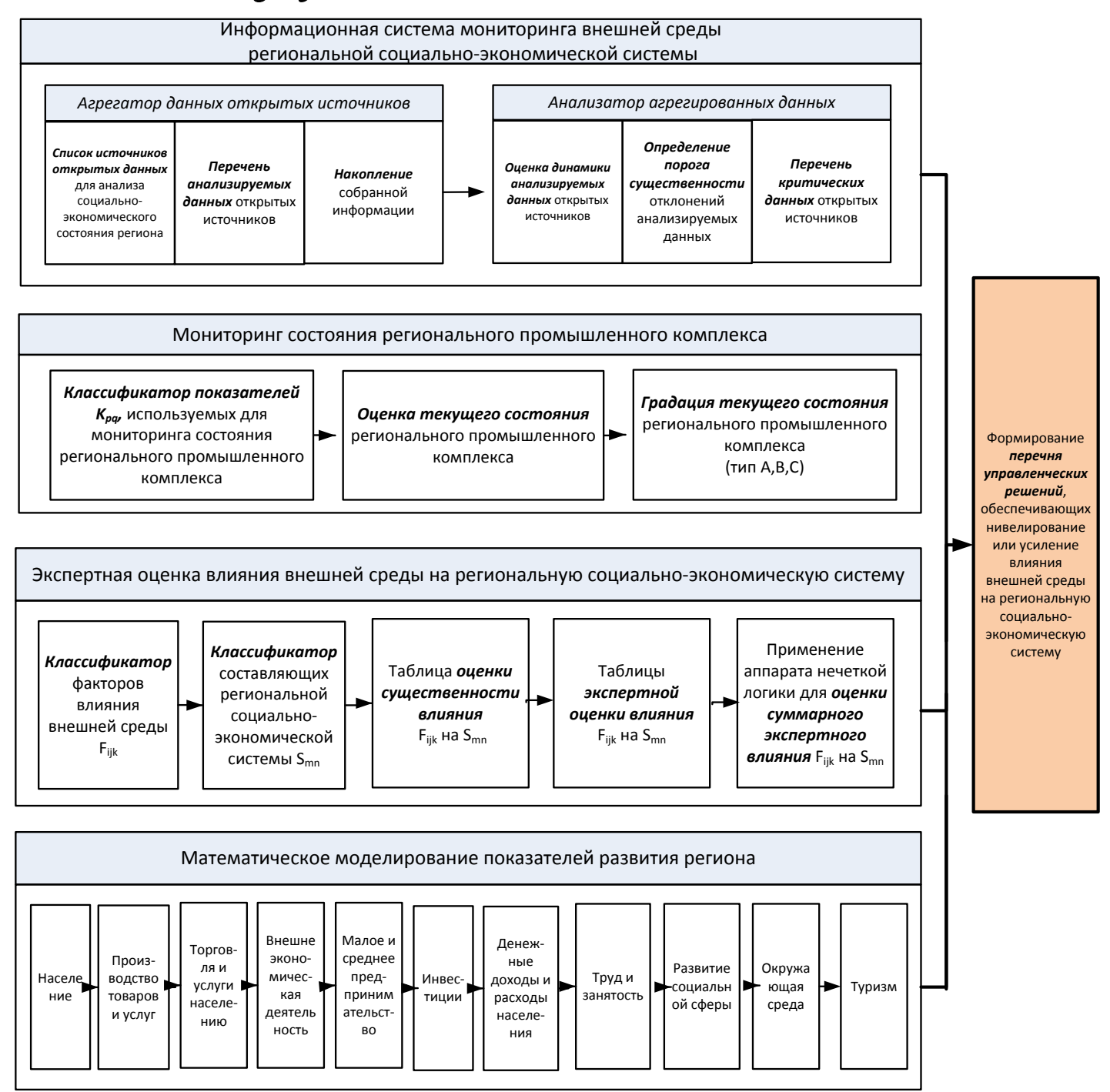

Рисунок 1. Место блока математического моделирования в концептуальной модели оценки влияния внешней среды на региональную социально-экономическую систему

Figure 1. The place of the block of mathematical modeling in the conceptual model for assessing the influence of the external environment on the regional socio-economic system

\section{Заключение}

Сформированная концептуальная модель влияния внешней среды на региональную социально-экономическую систему позволяет обосновать подход к созданию информационной советующей системы. Результатом работы автоматизированной системы будет являться формирование комплекса управленческих решений, позволяющих повысить качество управления на разных уровнях региональной власти.

\section{ЛИТЕРАТУРА}

1 Закон Брянской области от 21.12.2016 № 111-3 «Об областном бюджете на 2017 год и на плановый период 2018 и 2019 годов» в редакции Закона Брянской области от 27.04.2017 № 23-3 «О внесении изменений в Закон Брянской области «Об областном бюджете на 2017 год и на плановый период 2018 и 2019 годов»
Предлагаемая поддержка принятия управленческих решений будет основываться на независимой интеграции информационного мониторинга внешней среды, мониторинга регионального промышленного комплекса, экспертных оценках и результатах математического моделирования основных показателей развития региона, что обеспечит независимость и объективность комплексной оценки ситуации и формирования управленческих решений.

2 Постановление Администрации Брянской области от 01.04.2008 № 289 "Об утверждении регламента по разработке прогноза социально-экономического развития Брянской области на 2009 год и на период до 2011 года" URL: http://lawru.info/dok/2008/04/01/n526497.htm 
3 Основные показатели, представляемые для разработки прогноза социально-экономического развития Российской Федерации на период до 2019 года (для субъектов Российской Федерации) URL: http://www.bryanskobl.ru/docs/economy-forecast/form2p-2015.pdf

4 Averchenkov A.V., Averchenkova E.E., Gorlenko O.A., Miroshnikov V.V. Machine-building Enterprise Fuzzy Model as the Interrelated Factor Complex System// International Conference on Information Technologies in Business and Industry 2016. IOP Conf. Series: Journal of Physics: Conf. doi: 10.1088/17426596/803/1/012009. (SCOPUS: 2-s2.0-85016636779).

5 Averchenkova E.E., Averchenkov A.V., Kulagina N.A. Designing of the Information Advising System to Assess the Potential of Creation and Development of Cluster Agglomeration in the Industrial Complex of the Region// International Conference on Information Technologies in Business and Industry 2016. IOP Conf. Series: Journal of Physics. doi: 10.1088/17426596/803/1/012011 (SCOPUS: 2-s2.0-85018367471)

6 Аверченкова Е.Э., Гончаров Д.И. Обзор основных направлений формирования бюджета Брянской области на 2017-2019 гг. // Проблемы сохранения культурного наследия: материалы VI международного семинара, Брянск: ООО «Новый проект», 2017. 250 с. С. 162-165

7 Аверченкова Е.Э., Аверченков А.В. Концептуальная модель оценки влияния внешней среды на региональную социально-экономическую систему // Вестник БГТУ, 2016. № 9. С. 148-153

8 Treshchevsky Y.I., Litovkin M.V. Innovative activity in regions of Russia with various types of economic behaviour // Russian Journal of Agricultural and Socio-Economic Sciences. 2017. T. 64. № 4. C. 4-11.

\section{REFERENCES}

1 Zakon Brjanskoj oblasti ot 21.12.2016 \# 111 “Ob oblastnom bjudzhete na 2017 god i na planovyj period 2018 i 2019 godov” v redakcii Zakona Brjanskoj oblasti ot 27.04.2017 № $23 \mathrm{Z}$ “O vnesenii izmenenij v Zakon Brjanskoj oblasti "Ob oblastnom bjudzhete na 2017 god i na planovyj period 2018 i 2019 godov"

\section{СВЕДЕНИЯ ОБ АВТОРАХ}

Андрей В. Аверченков д.т.н., доцент, кафедра компьютерные технологии и системы, Брянский государственный технический университет, бул. 50-лет Октября, 7, г. Брянск, 241035, Россия, mahar@mail.ru

Е.Э. Аверченкова к.т.н., доцент, кафедра компьютерные технологии и системы, Брянский государственный технический университет, бул. 50-лет Октября, 7, г. Брянск, 241035, Россия,

Д.И. Гончаров к.т.н., доцент, кафедра компьютерные технологии и системы, Брянский государственный технический университет, бул. 50-лет Октября, 7, г. Брянск, 241035, Россия,

\section{КРИТЕРИЙ АВТОРСТВА}

Все авторы в равной степени принимали участие в написании рукописи и несут ответственность за плагиат

\section{КОНФЛИКТ ИНТЕРЕСОВ}

Авторы заявляют об отсутствии конфликта интересов.

ПОСТУПИЛА 10.04.2017

ПРИНЯТА В ПЕЧАТЬ 19.05.2017
2 Postanovlenie Administracii Brjanskoj oblasti ot 01.04.2008 \# 289 “Ob utverzhdenii reglamenta po razrabotke prognoza social'no-jekonomicheskogo razvitija Brjanskoj oblasti na 2009 god i na period do 2011 goda” Available at: http://lawru.info/dok/2008/04/01/n526497.htm

3 Osnovnye pokazateli, predstavljaemye dlja razrabotki prognoza social'no-jekonomicheskogo razvitija Rossijskoj Federacii na period do 2019 goda (dlja subektov Rossijskoj Federacii) Available at: http://www.bryanskobl.ru/ docs/economy-forecast/form2p-2015.pdf

4 Averchenkov A.V., Averchenkova E.E., Gorlenko O.A., Miroshnikov V.V. Machine-building Enterprise Fuzzy Model as the Interrelated Factor Complex System. International Conference on Information Technologies in Business and Industry 2016. IOP Conf. Series: Journal of Physics: Conf. doi: 10.1088/17426596/803/1/012009. (SCOPUS: 2-s2.0-85016636779).

5 Averchenkova E.E., Averchenkov A.V., Kulagina N.A. Designing of the Information Advising System to Assess the Potential of Creation and Development of Cluster Agglomeration in the Industrial Complex of the Region. International Conference on Information Technologies in Business and Industry 2016. IOP Conf. Series: Journal of Physics. doi: 10.1088/1742-6596/803/1/012011 (SCOPUS: 2-s2.0-85018367471)

6 Averchenkova E.E., Goncharov D.I. Overview of the main directions of formation of the budget of the Bryansk region in the 2017-2019 biennium. Problemy sokhraneniya kul'turnogo naslediya: materialy VI mezhdunarodnogo seminara [Problems of preservation of cultural heritage: materials of the VI international seminar]. Bryansk, New project, 2017. pp. 162-165. (in Russian).

7 Averchenkova E.E., Averchenkov A.V. A conceptual model of the impact of the environment on regional socio-economic system. Vestnik BGTU [Proceedings of BGTU]. 2016. no. 9. pp. 148-153. (in Russian)

8 Treshchevsky Y.I., Litovkin M.V. Innovative activity in regions of Russia with various types of economic behaviour. Russian Journal of Agricultural and Socio-Economic Sciences. 2017. vol. 64. no. 4. pp. 4-11. (in Russian).

\section{INFORMATION ABOUT AUTHORS}

Andrew V. Averchenkov doctor of technical sciences, assistant professor, computer technologies and systems department, Bryansk State Technical University, Bulvar 50-letiya Oktyabrya, 7, Bryansk, 241035, Russia, mahar@mail.ru

E.Je. Averchenkova candidate of technical sciences, assistant professor, computer technologies and systems department, Bryansk State Technical University, Bulvar 50letiya Oktyabrya, 7, Bryansk, 241035, Russia,

D.I. Goncharov candidate of technical sciences, assistant professor, computer technologies and systems department, Bryansk State Technical University, Bulvar 50-letiya Oktyabrya, 7, Bryansk, 241035, Russia,

\section{CONTRIBUTION}

All authors equally took part in writing the manuscript and are responsible for plagiarism

\section{CONFLICT OF INTEREST}

The authors declare no conflict of interest.

RECEIVED 4.10.2017

ACCEPTED 5.19.2017 